\title{
Healthy scientific environment promoted by society in India
}

\section{New Delhi}

SOME 107 prominent Indian scientists have formed the Society for Scientific Values (SSV) to promote integrity, objectivity and ethical values in the pursuit of science. Its chief aim is to evolve a healthy scientific environment "free from prejudices, bureaucratic formalisms, dishonesty, propagation of unsubstantiated research claims, suppression of dissent, showmanship, sycophancy and political manipulation".

The architect of this new movement is Dr Avtar Singh Paintal, director-general of the Indian Council of Medical Research (ICMR). Paintal says that the climate for scientific research in India has deteriorated since independence not because of inadequate research facilities or salaries for scientists but due to the lack of a healthy scientific environment. And the society's chief objective is to promote, by personal and collective effort, the ethics and norms of science "not only for the progress of science and technology in the country but also for national character".

The membership of SSV is open only to scientists with a clean record who are serious in promoting the society's ideals in the organizations in which they work. Members should never have plagiarized, made false claims or indulged in any kind of dishonest activity and should not have allowed their names to be included in publications in which they have not been actively involved.

The movement is gradually showing results, says Paintal. The eligibility criterion for authorship of papers is becoming widely accepted. The society has also persuaded the government to set up conference facilities for scientists: SSV prohibits

\section{Another large telescope to be sited on Hawaii's Mauna Kea?}

\section{Washington}

The National Optical Astronomy Observatories (NOAO) have chosen Mauna Kea in Hawaii as the prospective site for a huge new instrument consisting of four harnessed 8-m telescopes. The summit of this extinct volcano already houses one of the world's largest family of telescopes.

The National New Technology Telescope (NNTT) is the most ambitious of several current proposals espoused by various sections of the US astronomical community. Its four mirrors, each much larger than anything cast or ground so far, can be made to function as four separate telescopes, or as a single telescope or as an interferometer. They will be made by a technique called spin-casting, in which molten ceramic is rotated at a constant speed as it cools, so that the solidified mass takes on a parabolic curve. The blank is then figured by traditional grinding and polishing.

A 2-m mirror has been made by this method, and preparations for making a 3.5-m blank are almost complete. If successfully cast and polished, the $3.5-\mathrm{m}$ mirror will be used by the five-university Astrophysical Research Consortium for a new telescope at Apache Point, in the Sacramento Mountains of New Mexico.

The rival to Mauna Kea was Mt Graham, about 80 miles north-east of Tucson, Arizona. A comprehensive study was made of the scientific virtues of both sites, and Hawaii was preferred, according to NOAO, solely because its superior atmospheric conditions permit higher quality astronomical observations. But there was opposition to the Arizona site by environmental groups, particularly the Sierra Club. Mt Graham is remote and unspoiled, and has its own unique species of squirrel. Remoteness is, of course, a benefit for astronomy; observations at Kitt Peak are beginning to be marred by diffuse artificial lighting from Tucson and Phoenix.

The NNTT remains an astronomer's dream, with an expected cost of about $\$ 150$ million. Present National Science Foundation (NSF) spending, at a rate of $\$ 2$ million a year, supports only technological development, of which a good deal is needed. As well as using mirrors of unprecedented size, the NNTT will demand control systems and a mounting of largely untested abilities. Feasibility studies of these problems, says Pat Bautz at NSF, are aimed at producing a formal construction proposal within a few years. NSF will then decide whether to allocate funds from its regular astronomy budget.

These doubts are behind a feeling that it might be better to keep the NNTT and other big projects in abeyance for a few years while the privately funded Keck telescope gets under way. Construction of this device, a 36-element multiple-mirror telescope, will itself explore many of the technical problems the NNTT will have to face. The Keck project should be finished in 1992, and there is an understandable reluctance to commit federal funds before the verdict is in on that venture.

David Lindley

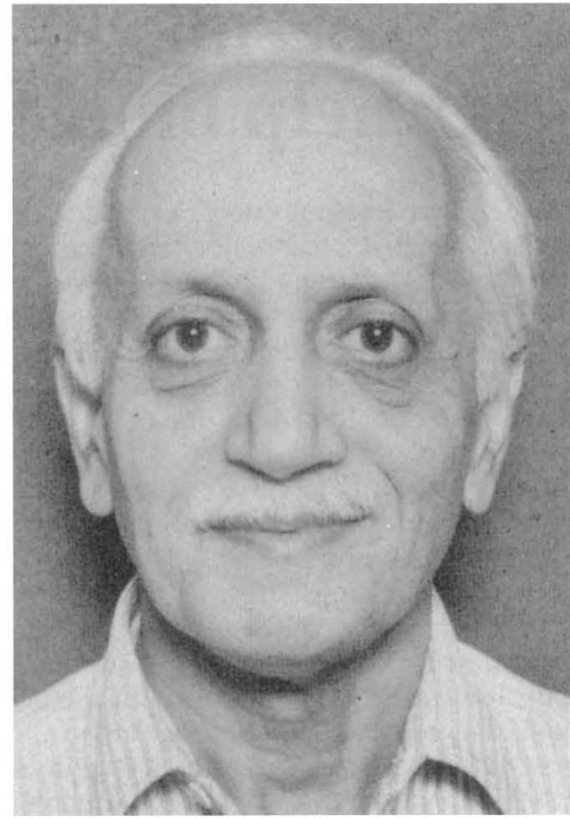

Dr Avtar Singh Paintal.

its members from attending conferences in expensive hotels beyond the reach of students and university scholars.

The practice of giving prizes year after year to the same group of politically influential scientists, who have long since ceased to do research, has become a scandal in India, and Paintal says that SSV hopes to put an end to this unhealthy tradition.

Paintal, has also redefined the goals of his own organization, ICMR. Although ICMR will continue to finance advanced research in medicine, it will chiefly be concerned with the application of existing technologies in solving India's health problems. Paintal's two priorities are the control of mosquitoes using the environmental approach and family planning using contraceptive pills.

According to Paintal, India has made a mistake by not promoting the pill. Fewer than 300,000 women in the whole of India at present use this method. At the instance of ICMR, the government is about to launch a major campaign advocating the use of the pill by women and of condoms by men.

On the control of malaria and filariasis, Paintal sees no need for new research. $\mathrm{He}$ says the only way to control these diseases is through elimination of the vector by environmental control, filling up ditches and improving sanitation. This approach has helped drastically to reduce the incidence of filariasis in Pondicherry and malaria in the Khera district of Gujarat. For the past 30 years, India has spent nearly half its health budget in the war against mosquitoes but without success. Paintal says that ICMR has shown that the battle can be won by simply cleaning up the environment instead of spending money on research on drugs, vaccines or insecticides.

K. S. Jayaraman 